\title{
Wnt- $\beta$-catenin signaling pathway inhibition by sclerostin may protect against degradation in healthy but not osteoarthritic cartilage
}

\author{
JIANG WU, LONG MA, LONG WU and QUNHUA JIN \\ Orthopedics Ward 3, The General Hospital of Ningxia Medical University, Yinchuan, Ningxia 750004, P.R. China
}

Received November 7, 2015; Accepted October 4, 2016

DOI: $10.3892 / \mathrm{mmr} .2017 .6278$

\begin{abstract}
The aim of the present study was to determine the regulation of sclerostin (SOST) in osteoarthritis (OA) and its effect on articular cartilage degradation. Human cartilage samples from healthy and OA subjects were assessed by Safranin O staining and immunohistochemistry. Primary chondrocytes were pre-incubated with $250 \mathrm{ng} / \mathrm{ml}$ SOST, $10 \mathrm{ng} / \mathrm{ml}$ interleukin-1- $\alpha$ (IL-1 $\alpha$ ) or a combination of the two. The effects of treatment on the Wnt- $\beta$-catenin signaling pathway and cartilage degradation were examined by reverse transcription-quantitative polymerase chain reaction and western blotting. SOST was detected in the cartilage focal area, demonstrating secretion by osteocytes and chondrocytes. SOST has been identified to inhibit the Wnt- $\beta$-catenin signaling pathway by binding to low-density lipoprotein-related receptors 5 and 6, and catabolic factors were decreased in healthy chondrocytes. However, SOST did not influence human OA chondrocytes. IL- $1 \alpha$ activated the Wnt- $\beta$-catenin signaling pathway and promoted cartilage degradation, which was inhibited by SOST in healthy and OA cartilage. The results of the present study suggested that SOST is important in maintaining the integrity of healthy, but not end-stage OA, cartilage.
\end{abstract}

\section{Introduction}

Osteoarthritis (OA) is a disease affecting the joints characterized by the thinning and disintegration of cartilage, synovial inflammation and subchondral bone remodeling (1). The primary feature of OA is the gradual loss of articular cartilage (2). OA progression is complex and involves numerous processes, including aggrecan and type II collagen degradation due to increased cleavage by activated proteolytic enzymes including matrix metalloproteinases (MMPs) and

Correspondence to: Professor Qunhua Jin, Orthopedics Ward 3, The General Hospital of Ningxia Medical University, 804 Shengli South Street, Yinchuan, Ningxia 750004, P.R. China

E-mail: jinqunhua0951@yeah.net

Key words: cartilage, osteoarthritis, sclerostin, Wnt- $\beta$-catenin, therapy a disintegrin and metalloproteinase with thrombospondin motifs (ADAMTSs) (3). Focal loss of articular cartilage in OA may be associated with alterations in the subjacent bone via modified load transmission and/or direct signaling between neighboring tissues (4).

Wnt signaling is critical for the regulation of adult bone turnoveenhancing this signaling pathway has been investigated as a potential therapeutic strategy for osteoporotic and inflammatory bone loss, to induce bone production and inhibit soluble antagonists (5). By contrast, increased Wnt- $\beta$-catenin signaling has been demonstrated to stimulate tissue degradation rather than formation in adult cartilage (6). Increased levels of $\beta$-catenin have been observed in chondrocytes at areas of cartilage degeneration (7), and it is upregulated in cartilage, particularly in the superficial cartilage zone (8). This may result in chondrocyte hypertrophy leading to cartilage damage (9). Furthermore, stimulation of chondrocytes with Wnt- $\beta$-catenin has been demonstrated to increase the expression levels of various factors involved in OA, including runt-related transcription factor 2 (RUNX-2), MMP-13, ADAMTS-4, and ADAMTS-5, facilitating cartilage matrix degradation (9).

Sclerostin (SOST), encoded by the Sost gene, is a secreted cysteine-knot protein of the differential screening selected gene abberative in neuroblastoma family, which acts as an antagonistic ligand for the Wnt coreceptors, low-density lipoprotein-related receptor (LRP)5 and LRP6, and an inhibitor of the canonical Wnt/ $\beta$-catenin signaling pathway (10). Although the alterations in SOST in human osteoarthritic cartilage have been described (11), the complex role of SOST during OA progression remains unclear. A previous study has demonstrated that SOST is additionally expressed by chondrocytes in mineralized cartilage (12). However, the therapeutic effects of SOST in OA cartilage remain controversial. Chan et al (13) demonstrated in vitro that increased chondrocyte SOST may protect against cartilage degradation in $\mathrm{OA}$, and Bouaziz et al (14) used SOST-knockout mice to reveal that the loss of SOST promotes OA in mice via $\beta$-catenin-dependent Wnt signaling pathways. However, Roudier et al (15) used SOST-knockout mice and an OA mouse model to demonstrate that SOST is expressed in articular cartilage, but its loss does not affect cartilage remodeling during aging or following mechanical injury. Whether SOST protects cartilage from degradation via inhibiting Wnt- $\beta$-catenin remains unknown. 
The present study therefore used healthy and OA chondrocytes to investigate the complex role of SOST in healthy and OA cartilage.

\section{Materials and methods}

Human samples. All human samples were obtained with the informed consent of patients, and with approval from the Ningxia Medical University Ethics Committee (Yinchuan, China). Human OA samples (OA group: $n=57$; female, 42; male, 15; age, 61.6 \pm 6.8 years) were obtained from patients undergoing total knee arthroplasty (TKA) for OA. Healthy human specimens (healthy group: $\mathrm{n}=6$; female, 2 ; male, 4;

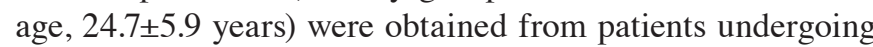
lower extremity amputation due to destructive injury. X-ray films of knees were used to determine whether patients had OA. Cartilage from the medial condylar, encompassing the maximal cartilage erosion focal area, was used for primary chondrocyte culture and subsequent mRNA and protein extraction. Medial tibial plateaus, encompassing the maximal cartilage erosion focal area, were isolated from patients prior to fixing in $4 \%$ paraformaldehyde for sectioning.

Histology. Knees were fixed in $4 \%$ paraformaldehyde for $24 \mathrm{~h}$ at $4^{\circ} \mathrm{C}$, decalcified in $0.5 \mathrm{M}$ ethylenediaminetetraacetic acid at room temperature for 21 days and embedded in optimum cutting temperature compound followed by paraffin. Serial 4- $\mu$ m-thick sagittal sections of the medial tibial plateau were obtained at three depths, at $50-\mu \mathrm{m}$ intervals. Sections were stained with Safranin O and cartilage degradation was determined using the modified Mankin scoring system (16). Samples were divided into three groups: Normal, mid-stage OA and end-stage OA. Paraffin sections for SOST immunostaining were first dewaxed and rehydrated, then antigen retrieval was performed with $0.1 \%$ trypsase (Beijing Solarbio Science \& Technology Co., Ltd.) and goat serum (Boster Bio-Engineering Ltd. Co., Wuhan, China) was used as a blocking antigen. Next, the slides were incubated with a primary rabbit anti-SOST antibody (cat. no. ab63097; Abcam, Cambridge, MA, USA) for $2 \mathrm{~h}$. Subsequently, biotinylated goat anti-rabbit IgG secondary antibody [cat. no. sv0002; Boster Bio-Engineering Ltd. Co.; 1:100, diluted in PBS containing 1\% bovine serum albumin (GibcThermo Fisher Scientific, Inc., Waltham, MA, USA)] was applied for $2 \mathrm{~h}$ at room temperature, followed by incubation for $1 \mathrm{~h}$ with horseradish peroxidase-conjugated antibody (cat. no. sv0002; Boster Bio-engineering Limited Company, Wuhan, China). Then the slides were colored with 3,3-diaminobenzidin (DAB) and stained with hematoxylin. Positive cells were stained yellow and counted under the light microscope (Olympus CX31, Olympus Corporation, Tokyo, Japan) on the tibial joint cartilage surface (magnification, x25) and expressed as a percentage of the total cells.

Ex vivo cartilage explant culture. Full-depth articular cartilage explants were isolated from the medial femoral condyle of patients undergoing TKA or amputation. Cartilage samples were cut into small pieces $\left(\sim 1 \mathrm{~mm}^{3}\right)$ and digested individually with $0.25 \%$ trypsin (Beijing Solarbio Science \& Technology Co., Ltd., Beijing, China) and collagenase II (Sigma-Aldrich; Merck Millipore, Darmstadt, Germany) to isolate chondrocytes.
As it is difficult to isolate chondrocytes from end-stage OA patients, due to limited cartilage on the bone surface, cartilage was not obtained from all OA patients. In total, 42 samples of OA chondrocytes $(n=42)$ and six samples of healthy chondrocytes were cultured. All chondrocytes were cultured in $20 \%$ fetal bovine serum (GibcThermo Fisher Scientific, Inc., Waltham, MA, USA) in Dulbecco's modified Eagle's medium (GibcThermo Fisher Scientific, Inc.) for 2 days to ensure cell adhesion. Chondrocytes from healthy subjects were subcultured once at a split ratio of 1:4 using trypsin, generating 24 culture dishes of healthy chondrocytes $(n=24)$. The medium was replaced every 3 days and activation of the Wnt- $\beta$-catenin signaling pathway was examined by incubating chondrocytes in the absence or presence of $10 \mathrm{ng} / \mathrm{ml}$ interleukin-1- $\alpha$ (IL-1 $\alpha$; PeproTech, Inc., Rocky Hill, NJ, USA) for 48 h. The effect of SOST was examined by addition of $250 \mathrm{ng} / \mathrm{ml}$ recombinant human SOST (PeproTech, Inc.) for $48 \mathrm{~h}$ in the absence or pres-

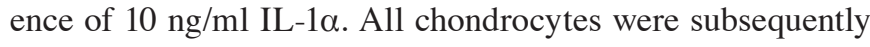
prepared for mRNA and protein extractions.

RNA extraction and reverse transcription-quantitative polymerase chain reaction $(R T-q P C R)$. RNA was extracted from human chondrocytes using the E.Z.N.A ${ }^{\circledR}$ Total RNA kit (Omega Bio-Tek, Inc., Norcross, GA, USA), and quantified with a NanoDrop 2000 spectrophotometer (Thermo Fisher Scientific, Inc.). Next, RT was performed using RevertAid First Strand cDNA Synthesis kit (Thermo Fisher Scientific, Inc.). The temperature protocol for RT was as follows: $25^{\circ} \mathrm{C}$ for $5 \mathrm{~min} ; 42^{\circ} \mathrm{C}$ for $60 \mathrm{~min}, 70^{\circ} \mathrm{C}$ for $5 \mathrm{~min}$ for termination as described in the kit manual. mRNA expression levels were quantified by qPCR using a SYBR ${ }^{\circledR}$ Green Master mix (Thermo Fisher Scientific, Inc.) and a LightCycler ${ }^{\circledR} 480$ (Roche Diagnostics, Basel, Switzerland). The temperature protocol for the reaction was as follows: $95^{\circ} \mathrm{C}$ for $10 \mathrm{~min}, 40$ cycles at $95^{\circ} \mathrm{C}$ for $15 \mathrm{sec}$, drawing melting curve at $65^{\circ} \mathrm{C}$ for $15 \mathrm{sec}, 60^{\circ} \mathrm{C}$ for $1 \mathrm{~min}, 95^{\circ} \mathrm{C}$ for $15 \mathrm{sec}$ and $60^{\circ} \mathrm{C}$ for $15 \mathrm{sec}$. The primers used are presented in Table I. Averaged quantification cycle $(\mathrm{Cq})$ values were normalized to the averaged $\mathrm{Cq}$ value of $\beta$-actin. Adjusted average $\mathrm{Cq}$ values were used to calculate relative expression vs. the control, using the $2^{-\Delta \Delta \mathrm{Cq}}$ method (17).

Western blot analysis. Chondrocyte proteins were extracted using a Whole Cell Lysis assay kit (Nanjing KeyGen Biotech Co., Ltd., Nanjing, China) and quantified using the bicinchoninic acid assay. Membranes were probed with the following primary antibodies: anti-SOST (1:1,000; cat. no. ab63097), anti- $\beta$-catenin (1:1,000; cat. no. ab6302), anti-MMP-13 (1:2,000; cat. no. ab39012) and anti-ADAMTS-4 (1:1,000; cat. no. ab84792; Abcam). Protein concentration was determined using the bicinchoninc acid protein assay kit (Boster Bio-engineering Limited Company, Wuhan, China). Equal quantities of protein $(20 \mu \mathrm{g})$ were separated by $10 \%$ SDS-PAGE and electroblotted onto a PVDF membrane (EMD Millipore, Billerica, MA, USA). The membrane was blocked in PBS containing 0.1\% Tween-20 (Beyotime Institute of Biotechnology, Inc., Haimen, China) and 5\% non-fat dry milk. The membrane was then probed with primary antibodiesfor overnight incubation at $4^{\circ} \mathrm{C}$. Horseradish peroxidase-conjugated rabbit anti-human secondary antibody (cat. no. BA1070; 1:2,000; Boster Bio-engineering Limited Company) was then 
A

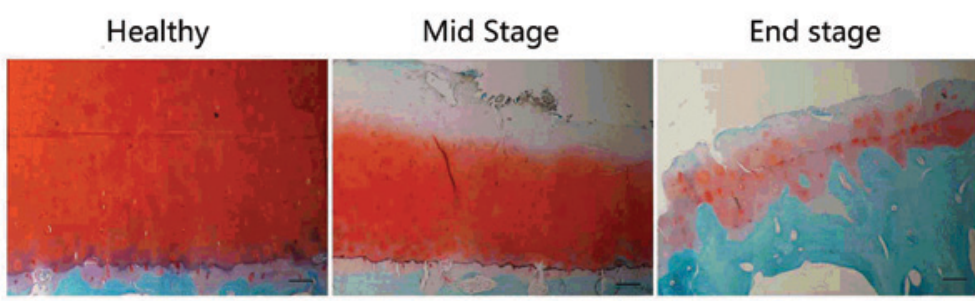

B

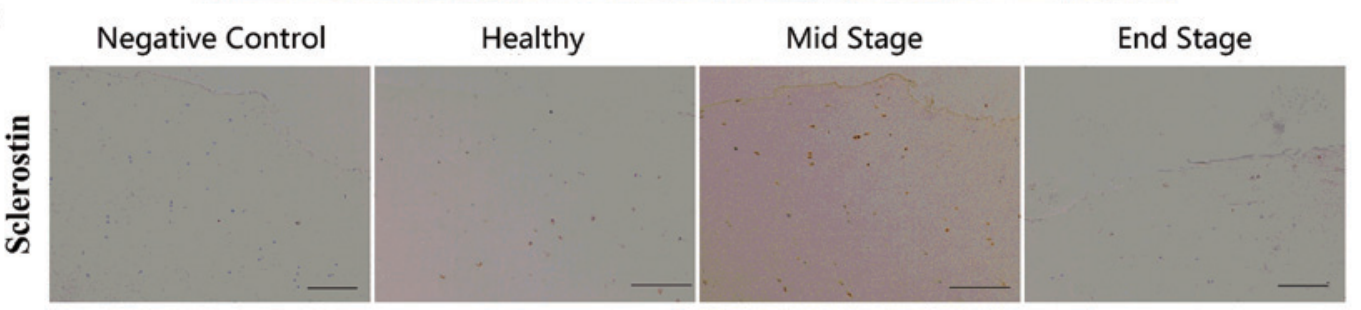

C

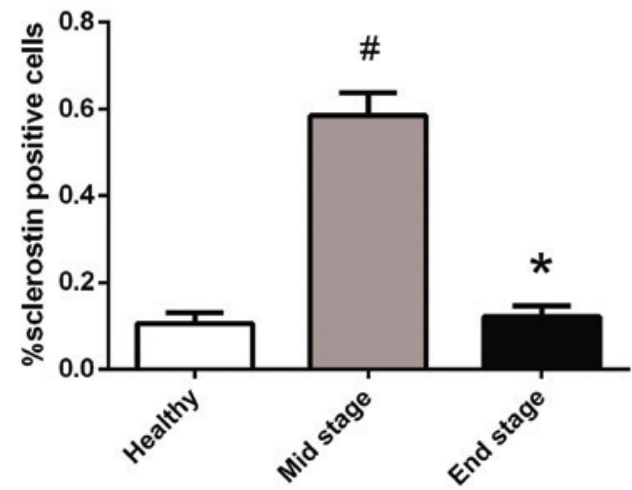

Figure 1. Expression of SOST in cartilage of OA patients and healthy controls. (A) Safranin O staining reveals the progression of cartilage lesions in the medial tibial plateau of healthy and OA cartilage. Scale bar=500 $\mu \mathrm{m}$. (B) Immunohistochemical analysis of SOST in calcified cartilage. Scale bar=200 $\mu \mathrm{m}$. (C) Percentage of SOST-positive cells in cartilage of tibial plateau. The percentage of SOST-positive chondrocytes in the mid-stage OA group was significantly increased compared with the healthy and end-stage OA groups. ${ }^{*} \mathrm{P}<0.05$ vs. healthy; ${ }^{*} \mathrm{P}<0.05$ vs. mid-stage. SOST, sclerostin; OA, osteoarthritis.

added for $1 \mathrm{~h}$ at $26^{\circ} \mathrm{C}$. Finally, the protein bands were detected using ECL solution (GE Healthcare Life Sciences, Shanghai, China) and images were captured using a FluorChem imaging system (Alpha Innotech, San Leandro, CA, USA). $\beta$-actin was used as a loading control. Unfortunately, western blot analysis was not performed on healthy group chondrocytes. Only six healthy cartilage samples were used, which were not subcultured to the third generation or cultured for a prolonged time in case of cytometaplasia and apoptosis. Therefore, not enough cells were obtained for western blotting and SOST, $\beta$-catenin, MMP-13 and ADAMTS-4 protein expression levels were detected only in OA human chondrocytes.

Statistical analysis. Data are presented as the mean \pm standard deviation, and were compared using SPSS software (version 22.0; IBM SPSS, Armonk, NY, USA). Two-way analysis of variance was used to compare the percentage of SOST-positive cells between healthy and OA cartilage groups. Alterations in the number of positively stained cells, and the fold change in gene expression are presented graphically as means with $95 \%$ confidence intervals. The protein expression levels in different groups were compared using a Student's $t$-test. As certain data, for example gene expression, were not normally distributed, treatment effects were assessed using the non-parametric Mann-Whitney $\mathrm{U}$ test. $\mathrm{P}<0.05$ was considered to indicate a statistically significant difference.

\section{Results}

Expression of SOST is not permanently increased in cartilage during OA progression. The Mankin score of the healthy group $(n=6)$ was $1.3 \pm 1.2$. Cartilage samples obtained from OA patients ( $n=57$ ) were divided into two groups, with scores for the mid-stage OA group $(n=37)$ being $7.7 \pm 0.9$ and the end-stage OA group $(n=20)$ being 13.0 \pm 1.2 (Fig. 1A). Immunohistochemical staining was performed to detect the expression of SOST at different stages of OA (Fig. 1B). Few SOST-positive stained chondrocytes were observed in the healthy and end-stage OA groups, with focal localization in the calcified cartilage and deep cartilage near the tidemark. However, the percentage of SOST-positive chondrocytes in the mid-stage OA group was significantly increased compared with the healthy $(\mathrm{P}=0.002)$ and end-stage OA groups ( $\mathrm{P}=0.001)$ (Fig. 1C). No differences were observed between the healthy and end-stage OA groups $(\mathrm{P}=0.48)$; therefore, SOST was not permanently increased during OA progression, instead increasing and subsequently decreasing. A high number of positively stained chondrocytes were observed in the calcified cartilage of the healthy group; however, only a few were present in the deep cartilage and none on the surface. Conversely, positively stained chondrocytes were widely distributed in the surface, deep and calcified cartilage in mid-stage OA cartilage, with low SOST expression in the calcified cartilage in end-stage OA samples. 
Table I. Primer sequences used for polymerase chain reaction analysis.

\begin{tabular}{lccccl}
\hline Gene & Accession no. & $\begin{array}{c}\text { Tm } \\
\left({ }^{\circ} \mathrm{C}\right)\end{array}$ & $\begin{array}{r}\text { Product } \\
\text { size }(\mathrm{bp})\end{array}$ & \multicolumn{1}{c}{ Forward } & Reverse \\
\hline SOST & NM_002427.3 & 60 & 126 & TTCTCCTTCGGGACCTCAAT & TCTCTCACCTCTGCCCATTC \\
$\beta$-catenin & NM_005099.4 & 58 & 122 & GCTTTGTGTCGTCTTGAACG & TCAGCAATCCCTTTCTCACC \\
LRP5 & AB257751.1 & 58 & 140 & TTCATCTACTGGACCGACTGG & TTGGTTCCGACGACCTTG \\
LRP6 & AB257752.1 & 59 & 114 & ATCAAGCACCAAAGGCACT & GTGGAAGGGCTGTTAGAAGAA \\
RUNX-2 & NM_001024630.3 & 60 & 100 & GACGAGGCAAGAGTTTCACC & GGTTCCCGAGGTCCATCTAC \\
MMP-13 & NM_001012329.1 & 60 & 129 & TATGACTATGCGTGGCTGGA & CCATTTGTGGTGTGGGAAGT \\
ADAMTS-4 & NM_005099.4 & 59 & 126 & GGCTGTGATCGCATCATTGG & CACATTGTTGTATCCGTACCTGA \\
ADAMTS-5 & NM_007038.3 & 59 & 172 & TGTGAAGAGACCTTTGGTTCC & TTCTGTGATGGTGGCTGAAG \\
COL2A1 & X02420 & 60 & 195 & CATTCATCCCACCCTCTCAC & TTCCTGTCTCTGCCTTGACC \\
$\beta$-actin & NM_001101 & 59 & 204 & TGACGTGGACATCCGCAAAG & CTGGAAGGTGGACAGCGAGG-3
\end{tabular}

Tm, annealing temperature; SOST, sclerostin; LRP, low-density lipoprotein-related receptor; RUNX-2, runt-related transcription factor 2; MMP, matrix metalloproteinase; ADAMTS, a disintegrin and metalloproteinase with thrombospondin motifs; COL2A1, collagen type II alpha 1 chain.

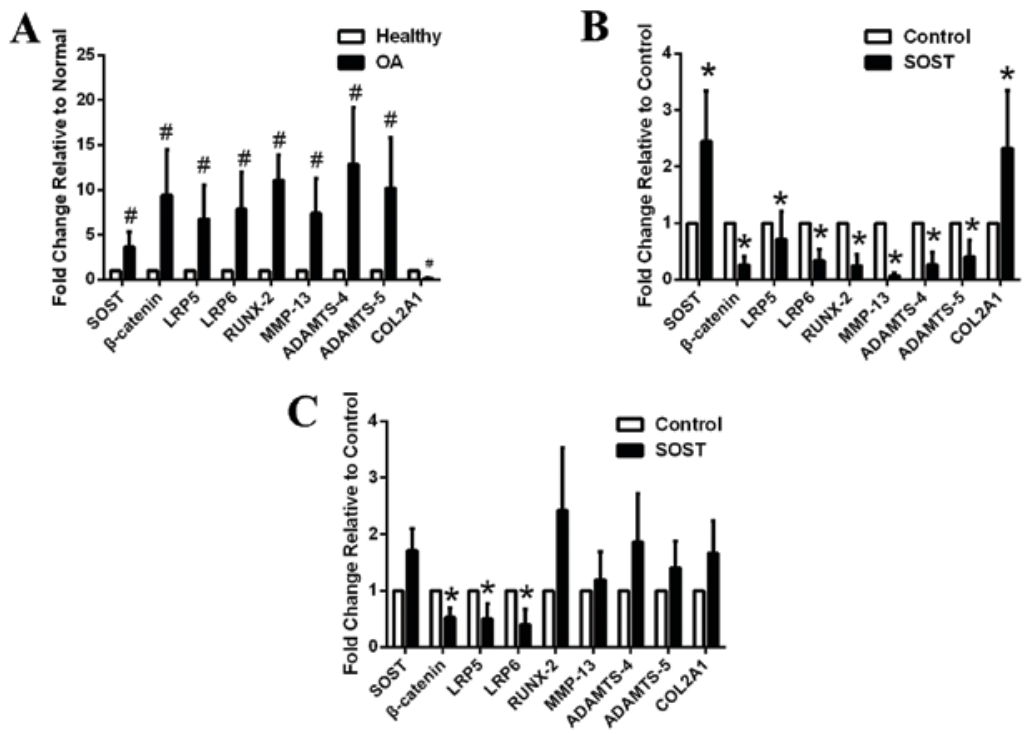

Figure 2. Expression levels of Wnt- $\beta$-catenin associated factors in healthy and OA chondrocytes incubated in the absence or presence of SOST. (A) The Wnt $-\beta$-catenin signaling pathway was overactivated in OA chondrocytes $(n=6)$ compared with healthy chondrocytes $(n=6)$. (B) SOST treatment increased the mRNA expression levels of the anabolic marker, COL2A1, but inhibited the mRNA expression levels of the Wnt signaling-associated factors, $\beta$-catenin, LRP5 and LRP6 and catabolic markers, RUNX-2, MMP-13 and ADAMTS-4,5 in healthy chondrocytes ( $\mathrm{n}=6)$. (C) SOST inhibited the mRNA expression levels of the Wnt signaling-associated factors, $\beta$-catenin, LRP5 and LRP6 in OA chondrocytes, but did not influence downstream factors ( $\mathrm{n}=6$ ). (D) Western blot analysis of OA chondrocytes incubated with SOST and/or IL-1 $\alpha .{ }^{\prime \prime} \mathrm{P}<0.05$ vs. healthy chondrocyte ${ }^{*} \mathrm{P}<0.05$ vs. control. OA, osteoarthriti SOST, sclerostin; LRP, low-density lipoprotein-related recepto; RUNX-2, runt-related transcription factor 2; MMP, matrix metalloproteinas; ADAMTS, a disintegrin and metalloproteinase with thrombospondin motif; COL2A1, collagen type II alpha 1 chain.

Wnt- $\beta$-catenin signaling is overactivated in OA chondrocytes. $\beta$-catenin $(\mathrm{P}=0.011)$, LRP5 $(\mathrm{P}=0.023)$ and LRP6 $(\mathrm{P}=0.012)$ mRNA expression levels were upregulated in $\mathrm{OA}$ chondrocytes, suggesting that the Wnt- $\beta$-catenin signaling pathway was overactivated and may be important in cartilage degradation (Fig. 2A). In addition, mRNA expression levels of the cartilage catabolic factors, RUNX-2 ( $\mathrm{P}=0.015)$, MMP-13 $(\mathrm{P}=0.015)$ and ADAMTS-4 $(\mathrm{P}=0.008)$, ADAMTS-5 $(\mathrm{P}=0.002)$ were upregulated, whereas the anabolic factor, COL2A1 $(\mathrm{P}=0.003)$ was downregulated, in the OA compared with the healthy chondrocyte group, thus accelerating cartilage matrix breakdown (Fig. 2A). Furthermore, SOST $(\mathrm{P}=0.021)$ mRNA expression levels were increased in the OA group, consistent with the results of the immunohistochemical analysis. Increased SOST expression may be a reaction to the activation of the Wnt- $\beta$-catenin signaling pathway and may inhibit the overactivated signaling pathway to maintain the integrity and normal structure of cartilage. If this is the case, SOST may be a potential therapeutic to delay OA progression.

SOST inhibits the Wnt- $\beta$-catenin signaling pathway in healthy and $O A$ chondrocytes, with beneficial effects observed in 

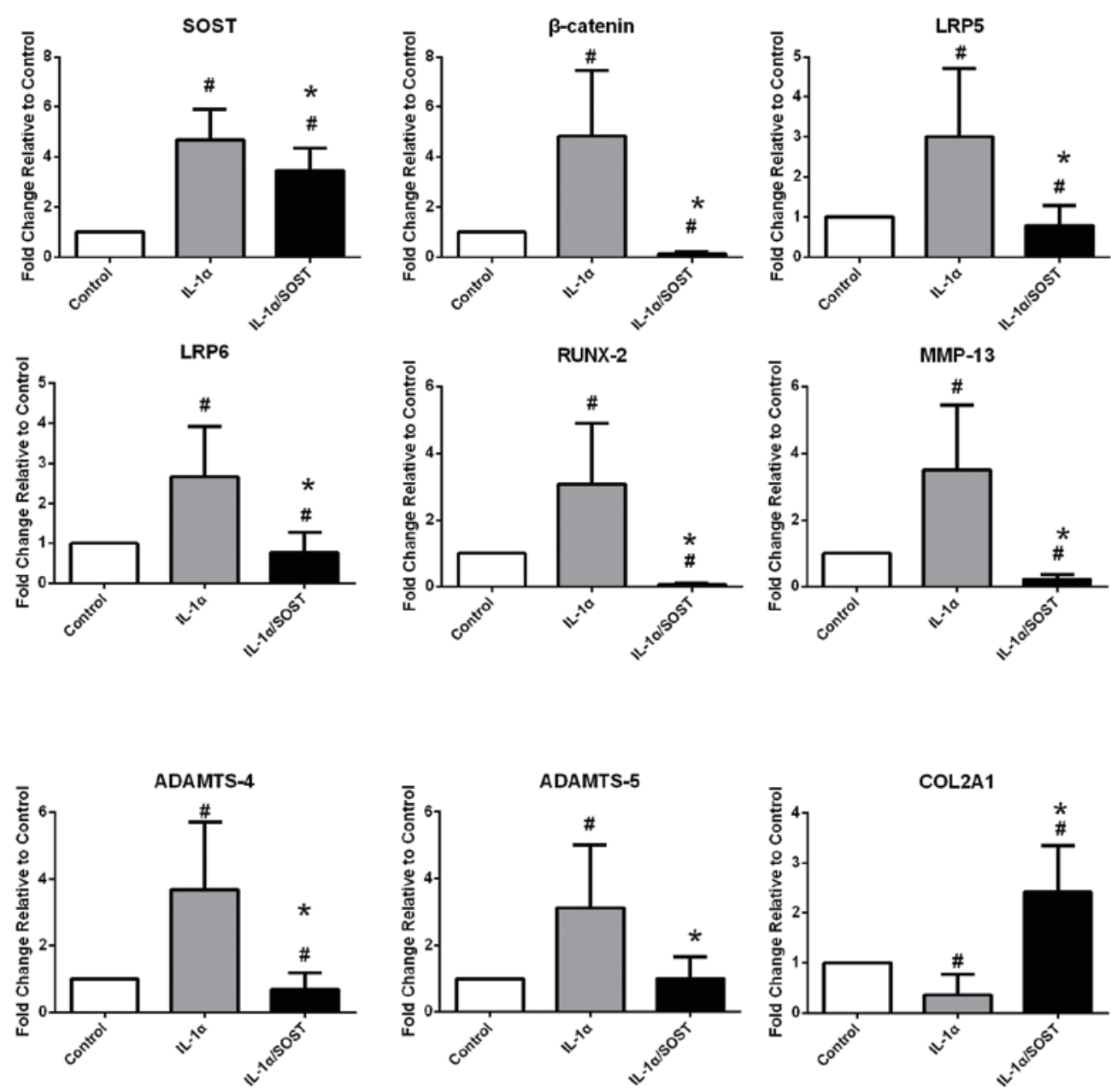

Figure 3. mRNA expression levels of Wnt- $\beta$-catenin-associated genes in healthy chondrocytes incubated with IL-1 $\alpha$ in the absence or presence of SOST. mRNA expression levels of the Wnt- $\beta$-catenin-associated factors, $\beta$-catenin and LRP5/6 and the catabolic markers, RUNX-2, MMP-13 and ADAMTS-4,5 were increased, whereas the anabolic marker, COL2A1 was decreased following IL-1 $\alpha$ treatment in healthy chondrocytes. These effects were inhibited by SOST $(n=6)$. The increasing of ADAMTS- 5 by IL- $1 \alpha$ was inhibited by SOST, but has no difference with the control group after the decreasing. ${ }^{~} \mathrm{P}<0.05 \mathrm{vs}$. control; "P<0.05 vs. IL-1 $\alpha$. IL-1 $\alpha$, interleukin-1- $\alpha$; SOST, sclerostin; LRP, low-density lipoprotein-related recepto; RUNX-2, runt-related transcription factor 2; MMP, matrix metalloproteinas; ADAMTS, a disintegrin and metalloproteinase with thrombospondin motif; COL2A1, collagen type II alpha 1 chain.

healthy chondrocytes only. SOST, as an inhibitor of the Wnt- $\beta$-catenin signaling pathway, may decrease the expression of downstream factors, including MMPs and ADAMTSs, to protect cartilage from degradation. Healthy (Fig. 2B) and OA (Fig. 2C) chondrocytes were incubated with $250 \mathrm{ng} / \mathrm{ml}$ SOST for $48 \mathrm{~h}$; this decreased $\beta$-catenin and LRP5/6 mRNA expression levels in the two groups. $\beta$-catenin (healthy group, $\mathrm{P}=0.012$ : OA group, $\mathrm{P}=0.022$ ), LRP5 (healthy group, $\mathrm{P}=0.026$; OA group, 0.0016) and LRP6 (healthy group, $\mathrm{P}=0.015$; OA group, $\mathrm{P}=0.013$ ). Therefore, $\mathrm{SOST}$ may inhibit the Wnt- $\beta$-catenin signaling pathway by binding to LRP5/6 in chondrocytes. In addition, SOST decreased RUNX-2 ( $\mathrm{P}=0.015)$, MMP-13 $(\mathrm{P}=0.006)$ and ADAMTS-4 $(\mathrm{P}=0.013)$, ADAMTS-5 ( $\mathrm{P}=0.015)$, and increased COL2A1 $(\mathrm{P}=0.002)$ mRNA expression levels in healthy chondrocytes (Fig. 2B). This indicated that SOST may assist in the maintenance of cartilage integrity and reduce cartilage damage. Although SOST treatment decreased $\beta$-catenin and LRP5/6 mRNA expression levels, it did not influence RUNX-2 ( $\mathrm{P}=0.065)$, MMP13 ( $\mathrm{P}=0.083)$, ADAMTS4 ( $\mathrm{P}=0.074)$, ADAMTS-5 $(\mathrm{P}=0.063)$ and $\mathrm{COL} 2 \mathrm{~A} 1(\mathrm{P}=0.068) \mathrm{mRNA}$ expression levels in OA chondrocytes compared with the control group (Fig. 2C).
This indicated that SOST may not have beneficial effects on OA chondrocytes despite decreasing $\beta$-catenin expression levels via binding to LRP5/6.

IL-1 $\alpha$ may regulate cartilage degradation via activation of the Wnt- $\beta$-catenin signaling pathway; this may be inhibited by SOST. Chondrocytes were incubated with $10 \mathrm{ng} / \mathrm{ml} \mathrm{IL-1 \alpha}$ for $48 \mathrm{~h}$ to simulate the inflammatory environment, determine the activation effect on the Wnt- $\beta$-catenin signaling pathway and quantify the effects of IL-1 $\alpha$ on chondrocytes. Following treatment with IL- $1 \alpha$ in the absence or presence of SOST, RT-qPCR was performed on healthy (Fig. 3) and OA (Fig. 4) chondrocytes and western blotting was performed on OA chondrocytes to detect protein expression of SOST, $\beta$-catenin, ADAMTS-4 and MMP-13 (Fig. 5). The mRNA expression levels of $\beta$-catenin ( $\mathrm{P}=0.002), \mathrm{LRP} 5(\mathrm{P}=0.004)$ and LRP6 ( $\mathrm{P}=0.014)$ in healthy (Fig. 3) were increased following treatment with IL-1 $\alpha$. Furthermore, mRNA expression levels of the cartilage catabolic factors, RUNX-2, MMP13 and ADAMTS4/5 were significantly increased, and the anabolic factor, COL2A1 was decreased, following treatment with IL- $1 \alpha$ in healthy (P-values were 0.015, 0.009, 0.022, 0.012; 

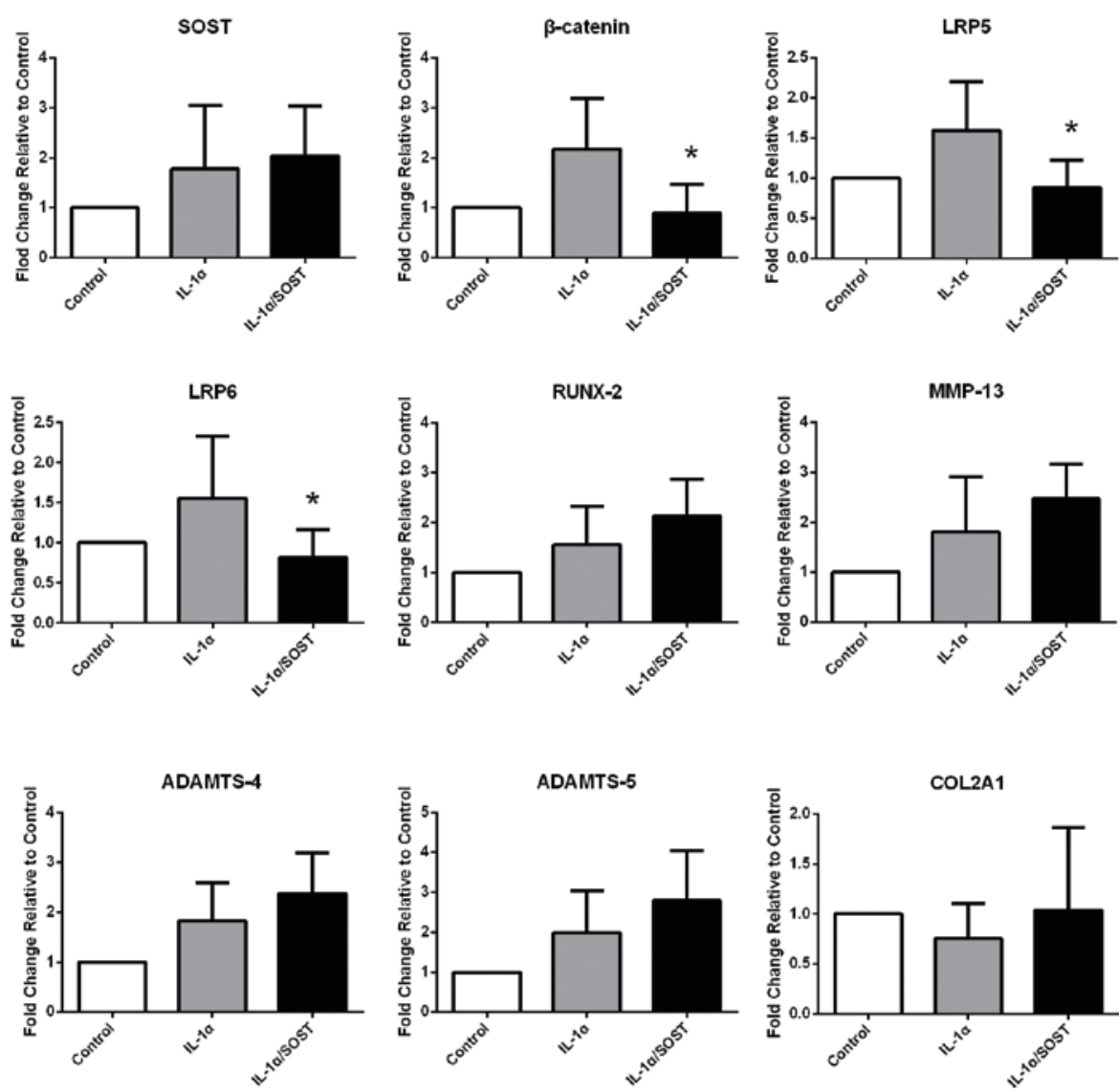

Figure 4. mRNA expression levels of Wnt- $\beta$-catenin-associated genes in OA chondrocytes incubated with IL-1 $\alpha$ in the absence or presence of SOST. mRNA expression levels of the Wnt $\beta$-catenin-associated factors, $\beta$-catenin and LRP5/6 were not influenced by IL-1 $\alpha$ in OA chondrocytehowever, the expression of these factors was inhibited by SOST. Catabolic and anabolic markers were not influenced by IL-1 $\alpha$ alone or with SOST ( $\mathrm{n}=6)$. ${ }^{*} \mathrm{P}<0.05$ vs. IL-1 $\alpha$. OA, osteoarthriti; IL-1 $\alpha$, interleukin-1- $\alpha$; SOST, sclerostin; LRP, low-density lipoprotein-related recepto; RUNX-2, runt-related transcription factor 2; MMP, matrix metalloproteinas; ADAMTS, a disintegrin and metalloproteinase with thrombospondin motif; COL2A1, collagen type II alpha 1 chain.

Fig. 3) but not OA (P-values were $0.62,0.53,0.71,0.53$; Fig. 4) chondrocytes. These results indicated that IL-1 $\alpha$ may accelerate the degradation of cartilage by activating the Wnt- $\beta$-catenin signaling pathway in healthy but not OA cartilage. Furthermore, chondrocytes exposed to IL-1 $\alpha$ demonstrated increased mRNA expression levels of SOST in the healthy $(\mathrm{P}=0.002)$ but not the $\mathrm{OA}$ group $(\mathrm{P}=0.56)$. Chondrocytes were treated with a combination of $250 \mathrm{ng} / \mathrm{ml}$ SOST and $10 \mathrm{ng} / \mathrm{ml} \mathrm{IL-1} \alpha$ to investigate whether SOST inhibited Wnt- $\beta$-catenin following overactivation. Compared with IL-1 $\alpha$ only, IL- $1 \alpha$ plus SOST treatment decreased $\beta$-catenin and LRP5/6 mRNA expression levels in healthy $(\mathrm{P}=0.002$, $0.012,0.012)$ and $\mathrm{OA}$ chondrocytes $(\mathrm{P}=0.022,0.002,0.015)$ and decreased RUNX-2, MMP-13 and ADAMTS4/5, and increased COL2A1 in healthy $(\mathrm{P}=0.023,0.009,0.013,0.016$; Fig. 3) but not $\mathrm{OA}(\mathrm{P}=0.68,0.57,0.55$; Fig. 4) chondrocytes. SOST therefore inhibited overactivation of the Wnt- $\beta$-catenin signaling pathway in healthy and OA chondrocytehowever, it did not decrease the expression of downstream catabolic factors to induce 'anti-catabolic' effects on OA chondrocytes.

\section{Discussion}

The etiology and progression of OA remain to be fully elucidated owing to the complexity of the diseastherefore, the role of SOST in cartilage and subchondral bone during degradation requires further investigation. Whether SOST protects cartilage from degradation remains controversial. SOST is typically expressed in osteocytes, particularly mature osteocytes, which are surrounded by mineralized matrix $(18,19)$. However, later studies have suggested that SOST is expressed by hypertrophic chondrocytes in calcified cartilage around the growth plate (20-23). Full-depth articular cartilage explants from the trochlear groove of ovine knee joints were used to examine the activation of IL- $1 \alpha$ in the Wnt- $\beta$-catenin signaling pathway; chondrocytes were revealed to express SOST, which is regulated by IL-1 (24). Increased SOST in human chondrocytes may inhibit the Wnt- $\beta$-catenin signaling pathway and downstream MMPs and ADAMTS in vivo, thus protecting cartilage from degradation (13). Bouaziz et al (14) demonstrated using destabilization of the medial meniscus (DMM) mice that SOST is only secreted by calcified matrix-embedded cells, and is increased during the development of OA but decreased in end-stage OA. In addition, this study demonstrated using SOST-knockout mice that loss of SOST accelerates degradation of cartilage in OA. However, Roudier et al (15) revealed using human articular cartilage that SOST is increased in OA cartilage, and that loss of SOST in the joint enhanced the bone mass of subchondral bonhowever, there was no difference between the cartilage 
A

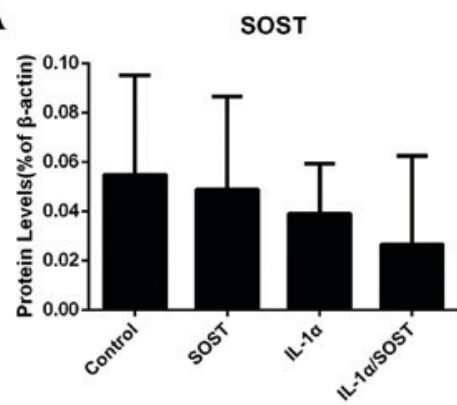

C

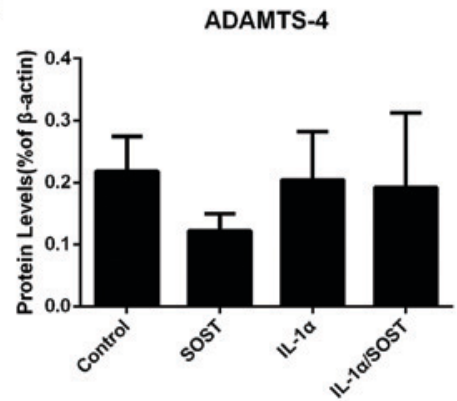

B

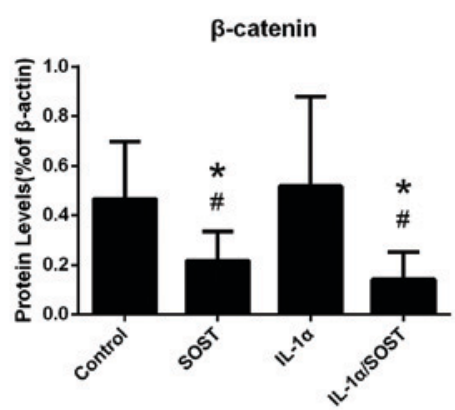

D

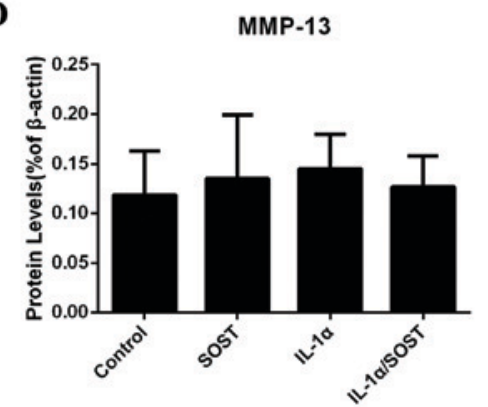

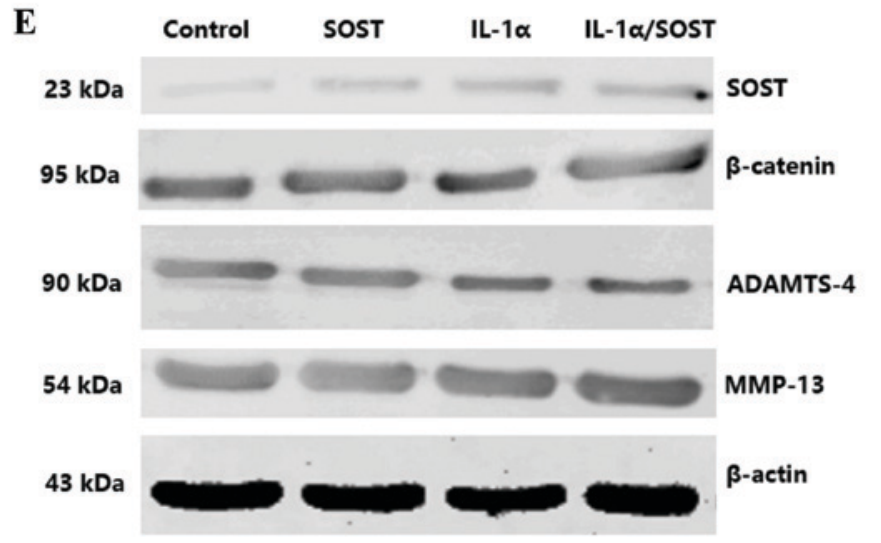

Figure 5. Protein expression levels of Wnt- $\beta$-catenin-associated genes in OA chondrocytes incubated with IL-1 $\alpha$ in the absence or presence of SOST. (A) Protein levels of SOST in OA chondrocytes was not influenced by IL-1 $\alpha(\mathrm{P}=0.76)$, SOST $(\mathrm{P}=0.43)$ and IL-1 $\alpha / \mathrm{SOST}(\mathrm{P}=0.17 ; \mathrm{n}=6)$ compared with control group $(\mathrm{n}=6)$. (B) Wnt- $\beta$-catenin-associated factors, $\beta$-catenin were influenced by SOST $(\mathrm{P}=0.035)$ and IL-1 $\alpha / \mathrm{SOST}(\mathrm{P}=0.024)$ in OA chondrocytes, but not influenced by IL-1 $\alpha(\mathrm{P}=0.15)$. (C) Protein levels of ADAMTS-4 in OA chondrocytes was not influenced by IL-1 $\alpha(\mathrm{P}=0.56), \mathrm{SOST}(\mathrm{P}=0.23)$ and IL-1 $\alpha / \mathrm{SOST}(\mathrm{P}=0.31 ; \mathrm{n}=6)$ compared with control group $(\mathrm{n}=6)$. (D) Protein levels of MMP-13 in OA chondrocytes were not influenced by IL-1 $\alpha(\mathrm{P}=0.13)$, SOST $(\mathrm{P}=0.21)$ and IL-1 $\alpha / \mathrm{SOST}$ $(\mathrm{P}=0.37 ; \mathrm{n}=6)$ compared with control group $(\mathrm{n}=6)$. (E) Western blot analysis of OA chondrocytes incubated with $\mathrm{SOST}$ and/or IL-1 $\alpha$. ${ }^{\sharp} \mathrm{P}<0.05$ vs. control; ${ }^{*} \mathrm{P}<0.05$ vs. IL-1 $\alpha /$ SOST. OA, osteoarthriti; SOST, sclerostin; LRP, low-density lipoprotein-related recepto; RUNX-2, runt-related transcription factor 2; MMP, matrix metalloproteinas; ADAMTS, a disintegrin and metalloproteinase with thrombospondin motif; COL2A1, collagen type II alpha 1 chain.

of SOST-knockout mice and DMM mice. Furthermore, increased SOST in cartilage did not affect cartilage remodeling during aging or following mechanical injury.

The present study demonstrated that SOST was expressed only in the focal area of calcified cartilage and deep cartilage adjacent to the tidemark in the medial tibial plateau of healthy and end-stage OA human articular cartilage. The expression of SOST was significantly increased in mid-stage OA and the positively stained chondrocytes were closer to the surface of cartilage compared with the healthy and end-stage OA groups. This is consistent with the study by Bouaziz et al (14) using DMM mice. SOST was not continuously increased in cartilage during the development of OA, instead first increasing and subsequently decreasing, which is in contrast to previous studies $(13,25,26)$. In early-stage OA, SOST in the cartilage may be secreted by osteocytes in the subchondral bone as a result of stimuli, including mechanical loading, but is not secreted by chondrocytes. Furthermore, in the present study almost every hypertrophic chondrocyte was positively stained. SOST therefore may not be secreted by healthy chondrocytes but by hypertrophic ones. In early-stage OA, chondrocytes in the cartilage did not express SOST; therefore, it must be secreted by osteocytes in the subchondral bone, from which it penetrates into cartilage via microchannels or vessels. Therefore, SOST-positive chondrocytes were observed only in the calcified cartilage. In mid-stage OA, the chondrocytes became hypertrophic and began to secrete SOST, resulting in a significant increase in SOST expression compared with 
early-stage OA (27). In end-stage OA, SOST expression was significantly reduced compared with mid-stage $\mathrm{OA}$. This is not consistent with some previous studies and further investigation is required to confirm these results.

In the present study, the Wnt- $\beta$-catenin signaling pathway was overactivated in OA chondrocytes, and matrix breakdown factors including MMPs and ADAMTSs were upregulated. Inhibiting the Wnt- $\beta$-catenin signaling pathway may decrease these mediators and therefore be a potential therapeutic approach for the treatment of OA. SOST has been identified to inhibit the Wnt- $\beta$-catenin signaling pathway by binding to LRP5/6 in healthy and OA chondrocytehowever, it decreased expression levels of cartilage catabolic factors only in healthy chondrocytes, with no beneficial effects observed on OA chondrocytes. This finding is inconsistent with previous studies in animal OA models $(13,26)$. It may be that SOST expression is affected by mechanical loading in osteoblasts in subchondral bone, and alterations of load bearing on cartilage may stimulate signal transduction between subchondral bone and cartilage. Due to the weight-bearing diversity across different areas of cartilage within the joint, SOST expression and its effect may vary between areas (28). As there is a marked difference in walking and limb alignment between humans and rodents, mechanical loading in the same area of cartilage within the joint may cause different consequences of gene expression, regulation and transduction of factors in the Wnt- $\beta$-catenin signaling pathway (29). In previous studies using rodent OA models, different experimental results may be due to a number of reasons. For example, using different joint cartilage regions for quantification due to the small scale and blurred boundaries of different parts of rodent joints, ignoring species specificity, in particular mechanical loading within the joint, and interactions of different signaling pathways. To avoid these errors, the present study used cartilage from the medial femoral condyle for chondrocyte isolation and primary culture to determine the effects of SOST on cartilage, and the medial tibial plateau for paraffin sectioning and immunohistochemistry to measure SOST expression in cartilage at different stages of OA. However, only six samples of healthy human cartilage were obtained. To generate sufficient chondrocyte numbers, cells were subcultured at a ratio of 1:4 to obtain 24 bottles of chondrocytes in the healthy group. Due to differing conditions in vivo and in vitro, chondrocytes were passaged only to the first generation in case of cytometaplasia and apoptosis. Consequently, not enough chondrocytes were obtained for western blot analysis, which may lead to inaccurate results within the present study. SOST may therefore only be a precautionary measure to prevent cartilage degradation, and not a potential therapeutic strategy to restore integrity or healthy cartilage structure during OA progression.

In addition, the route of injecting SOST may have a marked impact on whether SOST may be a potential therapeutic in OA. Intraperitoneal injection of SOST as a systemic drug delivery method would be convenient and effective. However, there is a very limited blood supply within cartilage tissue, although certain microchannels and micrangium penetrate calcified cartilage and the tidemark to deliver mediators between cartilage and subchondral bone. Whether SOST is small enough to access these channels remains unknown; therefore, the concentration of SOST in cartilage may not be enough to elicit an effect. Furthermore, SOST has been reported to inhibit osteoblast differentiation, proliferation and activity, resulting in reduced osteoblastic bone formation (30), which may lead to decreased subchondral bone stiffness and increased mechanical stress in cartilage. Intra-articular injection may have an advantage in maintaining the concentration of SOST; however, whether SOST permeates cartilage into the calcified region and subchondral bone remains unknown. It is currently difficult to state which drug-delivery method is more suitable.

To determine whether SOST inhibited overactivation of the Wnt- $\beta$-catenin signaling pathway in healthy and OA chondrocytes, chondrocytes were incubated with IL-1 $\alpha$ to activate Wnt signaling. IL-1 $\alpha$ treatment increased mRNA expression levels of $\beta$-catenin and downstream catabolic factors, including MMP-13 and ADAMTS-4/5, in healthy chondrocytes, and SOST inhibited this phenomenon. Notably, IL-1 $\alpha$ and SOST did not influence OA chondrocytes. This may be due to the reaction to the simulation of inflammatory factors of hypertrophic chondrocytes in OA not being as sensitive as healthy chondrocytehealthy chondrocytes may react to inflammatory stimuli and thus contribute to the maintenance of the normal structure and integrity of the cartilage, whereas hypertrophic chondrocytes may have lost this ability.

However, the results of the present study obtained by comparing SOST expression at different stages of OA and the incubation of chondrocytes with SOST ex vivo may not be accurate. All structures within the joint are affected and may interact during the progression of $\mathrm{OA}$, including the cartilage, subchondral bone and synovium. Chondrocytes and cells in the bone may react independently to identical environmental stimuli, leading to altered cell phenotypes in OA. Alternatively, cellular alterations in one cell type may have an affect on another cell type. Previous in vitro and in vivo studies have demonstrated that chondrocytes and osteoblasts influence each other $(31,32)$. Extensive research has demonstrated that cartilage and subchondral bone are not separate in the progression of $\mathrm{OA}$, therefore the progression of the disease should be considered in the context of the interaction of these two compartments $(33,34)$. Recently, molecular crosstalk between osteoblasts/osteocytes and chondrocytes has been demonstrated, revealing a previously unappreciated complexity (35). The dense subchondral vasculature in close proximity to the cartilage and the microchannels that infiltrate the subchondral mineralization region has been revealed to allow communication between bone and cartilage (36). Uncalcified cartilage may be observed dipping through the calcified cartilage into the bone and marrow spaces, which may provide a molecular diffusion pathway with potential nutritional, metabolic and biomechanical effects. In addition, as this interface is involved in OA, these areas may enable trafficking of humoral mediators between tissues (37). In the present study, no crosstalk was observed between subchondral bone and cartilage as it is challenging to simulate the physical and chemical environments within the joint. As alterations in one compartment may influence the other in $\mathrm{OA}$, the normalization of cells in one compartment may have beneficial effects on the other. The two compartments may 
subsequently influence each other to create a more favorable cycle, thus delaying the progression of OA.

Although the present study revealed that SOST benefited healthy but not OA chondrocytes, this does not mean that SOST may not influence chondrocytes, owing to the complexity of mediator translation between cartilage and subchondral bone. One possibility is that in the presence of SOST or as a result of pharmacologic inhibition, a compensatory molecule, for example another Wnt signaling inhibitor, is upregulated in the cartilagalternatively, the crosstalk between cartilage and subchondral bone may mask the effect of SOST inhibition. Further studies are required to clarify the complex role of the Wnt signaling pathway in OA and the interaction of SOST in the two compartments, which may contribute to an improved understanding of the pathogenesis and future therapies of human OA.

In conclusion, the results of the present study demonstrated that SOST is secreted by chondrocytes at various stages of $\mathrm{OA}$ and is not permanently increased in cartilage, first being increased and subsequently decreased. Furthermore, SOST inhibited the Wnt- $\beta$-catenin signaling pathway in healthy and OA chondrocytehowever, SOST downregulated catabolic factors to benefit only healthy chondrocytes. SOST may prevent cartilage degradation; however, it may be that SOST cannot restore the integrity or normal structure of cartilage during OA progression.

\section{References}

1. Bijlsma JW, Berenbaum F and Lafeber FP: Osteoarthritis: An update with relevance for clinical practice. Lancet 377: 2115-2126, 2011.

2. Felson DT: Developments in the clinical understanding of osteoarthritis. Arthritis Res Ther 11: 203, 2009.

3. Welgus HG: Stromelysin: Structure and function. Agents Actions Suppl 35: 61-67, 1991.

4. Karsdal MA, Leeming DJ, Dam EB, Henriksen K, Alexandersen P, Pastoureau P, Altman RD and Christiansen C: Should subchondral bone turnover be targeted when treating osteoarthritis? Osteoarthritis Cartilage 16: 638-646, 2008.

5. Hoeppner LH, Secreto FJ and Westendorf JJ: Wnt signaling as a therapeutic target for bone diseases. Expert Opin Ther Targets 13: 485-496, 2009.

6. Yuasa T, Otani T, Koike T, Iwamoto M and Enomoto-Iwamoto M: Wnt/beta-catenin signaling stimulates matrix catabolic genes and activity in articular chondrocytes: Its possible role in joint degeneration. Lab Invest 88: 264-274, 2008.

7. Zhu M, Tang D, Wu Q, Hao S, Chen M, Xie C, Rosier RN, O'Keefe RJ, Zuscik M and Chen D: Activation of beta-catenin signaling in articular chondrocytes leads to osteoarthritis-like phenotype in adult beta-catenin conditional activation mice. J Bone Miner Res 24: 12-21, 2009.

8. Blom AB, Brockbank SM, van Lent PL, van Beuningen HM, Geurts J, Takahashi N, van der Kraan PM, van de Loo FA, Schreurs BW, Clements K, et al: Involvement of the Wnt signaling pathway in experimental and human osteoarthritis: Prominent role of Wnt-induced signaling protein 1. Arthritis Rheum 60: 501-512, 2009.

9. Tamamura Y, Otani T, Kanatani N, Koyama E, Kitagaki J, Komori T, Yamada Y, Costantini F, Wakisaka S, Pacifici M, et al: Developmental regulation of Wnt/beta-catenin signals is required for growth plate assembly, cartilage integrity, and endochondral ossification. J Biol Chem 280: 19185-19195, 2005.

10. van Bezooijen RL, Roelen BA, Visser A, van der Wee-Pals L, de Wilt E, Karperien M, Hamersma H, Papapoulos SE, ten Dijke P and Löwik CW: Sclerostin is an osteocyte-expressed negative regulator of bone formation, but not a classical BMP antagonist. J Exp Med 199: 805-814, 2004.

11. Li X, Zhang Y, Kang H, Liu W, Liu P, Zhang J, Harris SE and Wu D: Sclerostin binds to LRP5/6 and antagonizes canonical Wnt signalin. J Biol Chem 280: 19883-19887, 2005.
12. Karlsson C, Dehne T, Lindahl A, Brittberg M, Pruss A, Sittinger $\mathrm{M}$ and Ringe J: Genome-wide expression profiling reveals new candidate genes associated with osteoarthritis. Osteoarthritis Cartilage 18: 581-592, 2010.

13. Chan BY, Fuller ES, Russell AK, Smith SM, Smith MM, Jackson MT, Cake MA, Read RA, Bateman JF, Sambrook PN and Little CB: Increased chondrocyte sclerostin may protect against cartilage degradation in osteoarthritis. Osteoarthritis Cartilage 19: 874-885, 2011.

14. Bouaziz W, Funck-Brentano T, Lin H, Marty C, Ea HK, Hay E and Cohen-Solal M: Loss of sclerostin promotes osteoarthritis in mice via $\beta$-catenin-dependent and -independent Wnt pathways. Arthritis Res Ther 17: 24, 2015.

15. Roudier M, Li X, Niu QT, Pacheco E, Pretorius JK, Graham K, Yoon BR, Gong J, Warmington K, Ke HZ, et al: Sclerostin is expressed in articular cartilage but loss or inhibition does not affect cartilage remodeling during aging or following mechanical injury. Arthritis Rheum 65: 721-731, 2013.

16. Moody HR, Heard BJ, Frank CB, Shrive NG and Oloyede AO: Investigating the potential value of individual parameters of histological grading systems in a sheep model of cartilage damage: The Modified Mankin method. J Anat 221: 47-54, 2012.

17. Livak KJ and Schmittgen TD: Analysis of relative gene expression data using real-time quantitative PCR and the 2(-Delta Delta C(T)) method. Methods 25: 402-408, 2001.

18. Poole KE, van Bezooijen RL, Loveridge N, Hamersma H, Papapoulos SE, Löwik CW and Reeve J: Sclerostin is a delayed secreted product of osteocytes that inhibits bone formation. FASEB J 19: 1842-1844, 2005.

19. Irie K, Ejiri S, Sakakura Y, Shibui T and Yajima T: Matrix mineralization as a trigger for osteocyte maturation. J Histochem Cytochem 56: 561-567, 2008

20. van Bezooijen RL, Bronckers AL, Gortzak RA, Hogendoorn PC, van der Wee-Pals L, Balemans W, Oostenbroek HJ, Van Hul W, Hamersma H, Dikkers FG, et al: Sclerostin in mineralized matrices and van Buchem disease. J Dent Res 88: 569-574, 2009.

21. Winkler DG, Sutherland MK, Geoghegan JC, Yu C, Hayes T, Skonier JE, Shpektor D, Jonas M, Kovacevich BR, Staehling-Hampton K, et al: Osteocyte control of bone formation via sclerostin, a novel BMP antagonist. EMBO J 22: 6267-6276, 2003.

22. Ellies DL, Viviano B, McCarthy J, Rey JP, Itasaki N, Saunders S and Krumlauf R: Bone density ligand, Sclerostin, directly interacts with LRP5 but not LRP5G171 V to modulate Wnt activity. J Bone Miner Res 21: 1738-1749, 2006.

23. Lin C, Jiang X, Dai Z, Guo X, Weng T, Wang J, Li Y, Feng G, Gao $X$ and He L: Sclerostin mediates bone response to mechanical unloading through antagonizing Wnt/beta-catenin signaling. J Bone Miner Res 24: 1651-1661, 2009.

24. Hwang SG, Yu SS, Ryu JH, Jeon HB, Yoo YJ, Eom SH and Chun JS: Regulation of beta-catenin signaling and maintenance of chondrocyte differentiation by ubiquitin-independent proteosomal degradation of alpha-catenin. J Biol Chem 280: 12758-12765, 2005.

25. Goldring MB and Goldring SR: Articular cartilage and subchondral bone in the pathogenesis of osteoarthritis. Ann N Y Acad Sci 1192: 230-237, 2010.

26. Lewiecki EM: Role of sclerostin in bone and cartilage and its potential as a therapeutic target in bone diseases. Ther Adv Musculoskelet Dis 6: 48-57, 2014.

27. Findlay DM and Atkins GJ: Osteoblast-chondrocyte interactions in osteoarthritis. Curr Osteoporos Rep 12: 127-134, 2014.

28. Bove SE, Calcaterra SL, Brooker RM, Huber CM, Guzman RE, Juneau PL, Schrier DJ and Kilgore KS: Weight bearing as a measure of disease progression and efficacy of anti-inflammatory compounds in a model of monosodium iodoacetate-induced osteoarthritis. Osteoarthritis Cartilage 11: 821-830, 2003.

29. Teichtahl AJ, Davies-Tuck ML, Wluka AE, Jones G and Cicuttini FM: Change in knee angle influences the rate of medial tibial cartilage volume loss in knee osteoarthritis. Osteoarthritis Cartilage 17: 8-111, 2009.

30. Baron R and Rawadi G: Targeting the Wnt/beta-catenin pathway to regulate bone formation in the adult skeleton. Endocrinology 148: 2635-2643, 2007.

31. Mohan G, Perilli E, Kuliwaba JS, Humphries JM, Parkinson IH and Fazzalari NL: Application of in vivo micro-computed tomography in the temporal characterisation of subchondral bone architecture in a rat model of low-dose monosodium iodoacetate-induced osteoarthritis. Arthritis Res Ther 13: R210, 2011. 
32. Findlay DM and Atkins GJ: Osteoblast-chondrocyte interactions in osteoarthritis. Current osteoporosis reports 12: $127-134,2014$

33. Amin AK, Huntley JS, Simpson AH and Hall AC: Chondrocyte survival in articular cartilage: The influence of subchondral bone in a bovine model. J Bone Joint Surg Br 91: 691-699, 2009.

34. Sanchez C, Deberg MA, Piccardi N, Msika P, Reginster JY and Henrotin YE: Osteoblasts from the sclerotic subchondral bone downregulate aggrecan but upregulate metalloproteinases expression by chondrocytes. This effect is mimicked by interleukin-6, -1beta and oncostatin M pre-treated non-sclerotic osteoblasts. Osteoarthritis Cartilage 13: 979-987, 2005.
35. Wang B, Zhou X, Price C, Li W, Pan J and Wang L: Quantifying load-induced solute transport and solute-matrix interaction within the osteocyte lacunar-canalicular system. J Bone Miner Res 28: 1075-1086, 2013.

36. Imhof H, Sulzbacher I, Grampp S, Czerny C, Youssefzadeh S and Kainberger F: Subchondral bone and cartilage disease: A rediscovered functional unit. Invest Radiol 35: 581-588, 2000.

37. Botter SM, van Osch GJ, Clockaerts S, Waarsing JH, Weinans H and van Leeuwen JP: Osteoarthritis induction leads to early and temporal subchondral plate porosity in the tibial plateau of mice: An in vivo microfocal computed tomography study. Arthritis Rheum 63: 2690-2699, 2011. 